\title{
The Failure Analysis and Treatment Measures of Relay Based on Power Systems
}

\author{
Jian Xu \\ School of New Energy \\ Shenyang Institute of Engineering \\ Shenyang, China \\ xujian@163.com
}

\author{
Zhuoran $\mathrm{Xu}$ \\ Shenyang Power Supply Branch \\ State Grid Liaoning Electric Power Supply Co. Ltd \\ Shenyang, China \\ xuzhuoran@163.com
}

\begin{abstract}
Power infiltrate every corner of social development, has become an integral part of the scientific progress and development , and improving quality of people's lives, and it is the era of progress closely. Electrical penetration in various fields has laid the power position in the social development. TV, refrigerators, computers and other electrical popularity, electricity began to soar. For power supply companies, powerful electricity load is a huge challenge on power supply work. We must take protective measures to ensure that residents and business can be normal power supply, these are a series of major problems. Routine maintenance of the relay, as well as maintenance, an important factor is to ensure electricity supply. This paper introduces the basic concepts, the role, composition and requirements about relay and analysis of the common faults and fault approach of the relay. These studies provide valuable experience for the future power system fault analysis and dealing with.
\end{abstract}

Keywords- infiltrate; integral; electricity; factor; analysis (key words)

\section{INTRODUCTION}

With the power system capacity is increasing, more and more widely, setting only the components of the system protection devices, can not prevent large-scale long-term serious accident occurred entire power system blackouts. So we must proceed from the overall power system, Fault element is the corresponding action of removal protection devices, the system will show what kind of conditions, what features will be lost when the system is stable, how to restore its normal operation and so on. The mission is to protect the system when a large power system uptime is destroyed, as far as possible to limit its scope to the minimum load is reduced to a minimum outage time[1]. In addition, machines, stoves, electric malfunction of any part of the power production safety are affected, especially the interaction and coordination of large units and large power systems is becoming a major issue of power production safety. Therefore, configuration protection and security automatic equipment design and manufacturing systems should consider varying bearing capacity machines, furnaces and other equipment, machine, furnace equipment should also take full account of the actual needs of the power system safe and economic operation. For security giant turbines, not only to maintain a good protection, but also research, promotion fault prediction techniques.

The term refers to the protection of power system protection technology and protection systems composed by a variety of protection devices. Relay is a complete system, including power system fault analysis, and implementation of the principle of protection, protection configuration design, relay operation and maintenance and so on. Work is complete circuit protection relay core functions, including the power to obtain information on the voltage, current transformer secondary circuit, through a variety of protection devices to trip coil with a set of equipment, the power supply and the necessary communications equipment.

Safety is very important to use electricity, while the relay is to ensure the safe conduct in the supply process The main role of power is the ability to meet the power supply, and how to measure the strength of an electric power companies, is to see whether it can continue to supply. Protection is to maintain the normal operation of the power supply process, thereby reducing accidents in the power supply process. If the presence of electrical accidents, and timely analysis, processing, look for the causes of the accident in a timely manner, to provide information for electrical safety maintenance personnel. In the process of electricity supply companies, the existence of protection can not be separated, and this is evident to all. Relay can decide whether to give full play, it depends on several conditions[2].

Protection, control, measurement, data communications integration in achieving protection of computerization and networking conditions, the protection device is actually a high-performance, multi-function computer, is an intelligent power system on the entire computer network terminal. It is available from the online power system operation and fault any information and data, and it can be any of the information and data protection elements it gets transmitted to the network control center or any terminal. Therefore, each computer protection device can not only complete protection function, but in the absence of a fault running Sui situation also complete the measurement, control, data communication functions, namely to achieve protection, control, measurement, data communication.

Research system failure and endanger the safe operation of the abnormal conditions of power, in order to investigate the anti-accident measures automated responses. 
Because in the course of its development has been mainly used to protect the relay contacts power systems and their components (generators, transformers, transmission lines), so that from the damage, so called protection. The basic task is: When the power system failure or abnormal condition in the shortest time possible and the minimum area, faulty equipment automatically removed from the system, or signaled by the staff on duty to eliminate the root causes of abnormal operating conditions, to reduce or avoid damage to the equipment and the impact on adjacent areas of supply.

\section{THE BASIC CONCEPT OF PROTECTION OF RELAY}

Research system failure and endanger the safe operation of the abnormal conditions of power, in order to investigate the anti-accident measures automated responses. Because in the course of its development has been mainly used to protect the relay contacts power systems and their components (generators, transformers, transmission lines, bus, etc.) to make it from damage, so called protection.

Reliability of the device,under its operating conditions, completed within a predetermined time and embodies all it has the ability[3]. Failure processing challenges to its work element means data reliability and apparatus of a direct relationship between the case means operating expenses scenario determines the reliability of the standard evaluation criteria, the reliability of the relay device, allows working under conditions Further failure, relay device should not have refused action, under conditions other protective action should not, should not relay device malfunction, regardless of tripping or malfunction occurs, will cause harm to the power system devices, due to structural load capacity of the power system there are differences, the nature of damage and damage effects tripping and malfunction caused also vary. Tripping and malfunction of maintenance measures, often contradictory. Adequate turning space within the system, a number of transmission lines, the close links between the various systems, a close link between the power source and the load, this state if the relay device malfunction occurs, it will direct the transmission line transformers and generators removal, so the smaller the damage caused. In the state of transmission lines and generators, transformer failure, tripping relay device, it will cause huge damage to the power system, destabilizing power system, damage electrical equipment. In this state, the relay device does not refuse to move relative to the malfunction, has a more significant; however, if the rotation is not sufficient space within the system, the power supply and the system carries contact does not close, if the malfunction occurs at this time phenomenon, transmission lines and transformers are cut will lead to failure of the power supply load, or damage to the whole state. Losses is difficult to predict. At this event tripping, faults can be completely removed, therefore, in this case, as opposed to not refuse to move, to take to enhance the means will not malfunction even more important[4].
To complete the relay task, in addition to external protection devices must work through a reliable protection work correctly circuit, faulty components to complete the circuit breaker tripped, not the normal operation of the power system components, or alerts normal operation without action task.

General work protection circuit comprising: a primary current through the power device, the voltage is linearly converted to other secondary devices used for protection of current, voltage, and to isolate the primary equipment and secondary equipment devices, such as current, voltage transformer and cable protection devices, such as connected; connecting cable circuit breaker trip coil and exports between the protection device, which indicates the case of signal equipment protection action; protection devices and tripping signal circuit device operating power supplies[5]. Protection devices block diagram of the principle agenciess is shown in Fig .1.

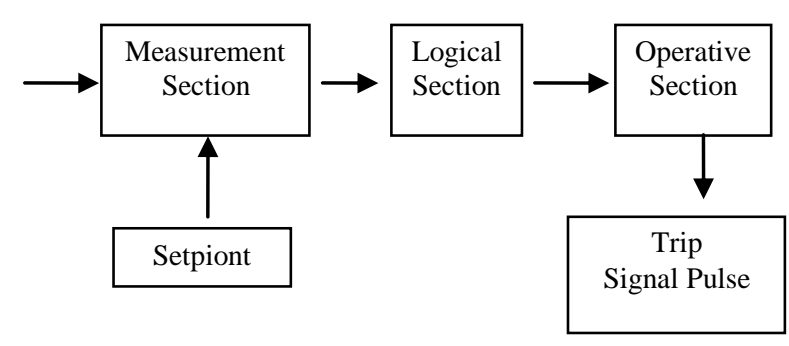

Figure 1. Protection devices block diagram of the principle agenciess

\section{POWER SYSTEM RELAY ROLE, COMPOSITION AND REQUIREMENTS}

In the modern power system, it has a lot of relating to the safety and economic operation optimization problems which can be attributed to the solution of optimal power flow. The power flow transmission capacity constraint is an important constraint of optimization problems, and in some cases may even become the bottleneck constraints of safety and economic operation of the system. Therefore, the study of wind power optimization method for power flow control problem is a better direction [8].

Based on optimal control trend of wind power mainly has two kinds of patterns:

\section{A. The role of relay}

Hysteresis generating element protection, protection relay unit independently, automatically identify the failed component, and then removed, the protection of the overall security. Components will not continue to cause damage and reduce the impact of power outages surface. Damage to protected components produced unusual situation occurs, the relay will respond quickly, according to the transmitted signal, reducing its load, then a trip, this time, do not need to issue a protective action immediately, to the extent of damage to components, take the length of time necessary to ensure its security, stability, stop. Target relay device 
voltage and current can be obtained in a timely manner of digital, enabling the reaction of power system operation is important for a power system of regulation[6].

\section{B. The Composition and requirements of relay}

Protection devices including some input, measurement, logic, output execution[7]. Input refers to the signal preprocessing, on-site to ensure that appliances can effectively detect physical quantities, such as isolation, level shifting, low-pass filtering. Need to output the measurement signal output order, size, logic state, properties and the like, after a reasonable calculation, in accordance with a fixed relationship between the final execution of the logic operation, shift by the final relay device outputs a logic signal having a fast, active, select resistance, flexible and reliable features, specific objects can be removed to protect the rest of the circuit to achieve the goal of reducing the harm, no part of the fault circuit produced can also continue to run, improve work efficiency, reduce the spread of the scope of failure to achieve alternate equipment, automatic reclosing automatically put into effect. While the response action failure to complete the first time, the situation is not normal to sensitive and efficient manifestation of a very high reliability and stability.

\section{Abnormal or defective}

Relay operation found abnormal or defective, in addition to strengthening surveillance, the right can cause malfunction of protection back its export plate, then contact following the security staff. If these unusual circumstances, should be timely exit:

Bus differential protection. In issuing the "mother poor exchange disconnection", "mother disappears DC voltage difference" signal; when the bus differential current imbalance is not zero; no dedicated bypass bus lines mother of the switch string generation and recovery operations in the switching operation.

High-frequency protection. Periodic channel test parameters do not meet the requirements;; when the DC power supply device failure or disappear abnormal channel can not be reset when the signal is issued; parent process next-generation line switch operation.

Distance protection. When the PT quit running or threephase voltage circuit break; under normal circumstances, to help the magnetic current is too large, too small; allowable current when the load current exceeds the corresponding segment protection.

Computer protection. Total warning lights, while the four protection (high frequency, distance, zero sequence, comprehensive weight) when one warning lights, exit corresponding protection; if two CPU failure, should withdraw from all the protection of the device; alarm plug all lights are not bright, If the power light is off, the DC disappear, should withdraw export plate, and then restore the DC power input; total warning lights and call lights, and print display CPU $\times$ ERR signal, such as CPU normal, indicating that the interface between the CPU and the communication circuit protection abnormal exit polling switch CPU processing, if the signal can not be reset, indicating CPU has a fatal flaw, it should protect the export exit inspection plate and disconnect switch handle[8].

Gas protection. When you need to open the respiratory system or put the oil discharge valve plug, or clean breathers;; refueling operation in the transformer when the oil filter or replace silicone; submersible pump or oil cooler (radiator) put the oil back into the maintenance load time Tap oil on the road when someone work .

\section{RELAY COMMON FAULTS AND SOLUTIONS}

\section{A . Relay common faults}

Improper selection switch protection equipment. Since the majority of high load, dense areas are required for the establishment of distribution switching stations, which supply mode that is substations, switching stations, distribution transformers, switch to select effective protection devices also have important significance, yet some switching station has the ability to automate protection can be taken to protect the load switch on the power system.

Fault current transformer saturation.Due to the increasing load terminal equipment, to produce a short circuit current generated also increases, so the relay device by saturation effects from the current transformer, also increased. Short circuit occurs when the system is close to the location of the terminal equipment, the current generation will be close to or more than 100 times the rated current of a single current transformer, the current transformer error and the short-circuit current is proportional to maintain multiple relationships, prevent action also due to the current quick break, when reduced sensitivity. Definite time overcurrent protection device will cause a short-circuit current of the current transformer saturation, resulting in its perception of the secondary current is close to zero, then the action will not be issued. Position in the export line, overcurrent protection device refused action, thereby promoting the occurrence and distribution of imported line protection action, will cause the entire power system blackouts.

\section{B. Solutions}

Measures to ensure the normal operation of power system protection.

In order to ensure the normal operation of the power system, to ensure the smooth conduct of the entire work, the need to further improve the system, based on the work of the required content protection and rational targeted want to specify an effective management system, and promoting coordination of conservation efforts to expand science configuration of personnel, and the rational allocation of tasks to staff and improve efficiency. Active protection equipment operation and maintenance, periodic calibration, defect treatment, accident analysis, etc., strict examination by computer management system, follow-up examination, the implementation of incentive measures to achieve secondary equipment condition monitoring[9].

Common treatment failure protection

Replacement method: Using replace the faulty component elements intact, then the fault is determined, in favor of rapid narrowing the scope of failure.

Reference method: the stability of the device parameter to control the parameters of the faulty device, then find 
fault lies, wiring mistakes or check the setpoint value and the expected value out of the test is large, this method is widely used, after the replacement, repair faulty components, after the secondary wiring, still there is a fault, then the need for control wiring similar device for repair, testing for performance, you can replace the relay scale, or with reference to the same table and the same relay circuit[10].

Short Connection: scope for further identification of failure, which uses short circuit to be short a period to detect the existence of this short range failures. Switching relay does not operate normally, electromagnetic lock failure, current open circuit failure has effectively applied, you can quickly switch to make a determination of the contact is intact.

\section{CONCLUSION}

Economic growth needs to be based on the construction of power, people want to have a high quality of life, then the power protection system is absolutely essential to existence. Relay is powered during escort, speed upgrade for the protection of the technical level, on top of the scale of construction must also be improved.Visible relay device huge role and influence in the protection of power systems, along with economic development and urban development, all walks of life to work on the power system power requirements are rising, the power system-related science and technology, scientific methods are constantly upgrading, rising, many problems will be followed by protection devices, the emergence of new changes in the new forms of protection to carry out the work, the need to constantly towards digital, information technology, network development, advancing toward the intelligent, which requires the relay staff constantly learning new knowledge, new technology, attention to maintenance of protection devices, the promotion of new technology applications, to enrich and improve the power system-related technology industry the theory provides a steady stream of power.

\section{ACKNOWLEDGMENT}

The authors gratefully acknowledge the contribution of co-workers and reviewers' comments.

\section{REFERENCES}

[1] Y.L Wang, "System reliability power protection devices running explore," Grid technology, vol.28,pp.83-85, July 2004

[2] W.Wang, “ Reliability Theory inrelay protection,”Sichuan. Elec. Pow.Tech, vol. 1,pp.23-27, May 2006

[3] X.Z.Wang and X.Chen.,"Protection coordination of distributed generation and distribution," Relay, vol. 9,pp.27-31, July 2009

[4] Y.Sun,N.S.Hang,and R.Q.Li, "Reliability and protection of Dynamic Performance Simulation,” Pow.Sys.Eng, vol.22,pp.34-39, April 2010

[5] Z.H.Dai and Z.P.Wang, "Summary protection reliability study," Pow.Sys.Pro.Con, vol.15,pp.12-16, June 2010

[6] Y.Lv, H.B.Sun, B.M.Zhang, and L.H.,Ma, "The development of online protection intelligent early warning system," Auto.Elec.Pow.Sy,vol.4,pp.34-38, May 2006

[7] Y.Z.Ge, "Progress protection technology," Relay,vol.1,pp.6-10, May 1998

[8] R. Brown, “ Review on Development of new protection,’Elec.Pow.Aut. Equ, vol.5,pp.34-38, May 2000

[9] Y.P.Feng, "Substation operation Problems and Countermeasures," Chi.New.Tec. New. Produ, vol.24, pp.4-8, April 2011

[10] Z.X.Shi, "On the relay in the substation reliability applications running," IEEE .Trans. Pow. Del, vol.18, pp.10-16April 2012 